\title{
Dijkstra Shortest Path Algorithm using Global Positioning System
}

\author{
Pooja Singal \\ DCSA, MDU, Rohatk
}

\author{
R.S.Chhillar \\ HOD, DCSA, MDU, Rohatk
}

\begin{abstract}
Dijkstra's Algorithm is used to find the shortest path from one node to another node in a graph.Dijkstra's algorithm is also known as a single source shortest path algorithm. It is applied only on positive weights. In this paper, Global Positioning System is used for adding a new functionality in Dijkstra's algorithm. In this paper, using Global Positioning System the position parameter is added in the Dijkstra's algorithm. From this current position is retrieved at any point. By using this current position, the distance can be determined from one node to another node. The shortest path can also find out using this distance. For this an algorithm is proposed.
\end{abstract}

\section{Keywords}

Dijkstra Shortest Path Algorithm (DSPA), Global Positing System (GPS), position, distance, node.

\section{INTRODUCTION}

In this we discuss two things. These are:

\subsection{Dijkstra Algorithm}

Dijkstra's algorithm is invented by Dutch computer scientist Edsger Dijkstra in 1956 and published in 1959, is a graph based searching algorithm that solves the single source shortest path problem. It is applied only on positive weights graphs. This algorithm is often used in routing. Dijkstra's algorithm is used for finding the shortest path with minimum cost.

"For example:-Let vertices in a graph the cities \&edges which link these vertices are the driving distances from one city to another city. DSPA is used to find the shortest route from one city to another with minimum cost. It solves only the problems with nonnegative costs, i.e., $C_{i j}>=0$ for all $(i, j)$ belongs to $E$, Where $\mathrm{C}$ is the cost $\& \mathrm{E}$ is the edges for a graph.

\subsection{Global Positioning System (GPS)}

GPS is a satellite based system that can be used in navigation to locate the positions anywhere on the earth. GPS is designed \& operated by U.S. Department of Defense (DOD).GPS consists of satellites, control \& monitor stations and GPS receivers. GPS receivers take information which is transmitted from the satellites and uses triangulation to calculate a user's exact location. GPS is used in a variety of ways:

- To determine the position of locations.

- To navigate from one location to another.

- To create digitized maps.

- To determine the distance between two points.

\subsubsection{Working of GPS}

The basis of GPS is a constellation of satellites that are continuously orbiting around the earth. These equipped with atomic clocks \& transmit radio signals that contain their exact location, time and other information. The radio signals which are transmitted from the satellites are monitored \& corrected by control stations which are sent back to satellites using ground antenna. The radio signals from satellites are picked up by the GPS receiver. A GPS receiver needs only 3 satellites to plot a rough, 2D position, which will not be very accurate. Ideally, 4 or more satellites are needed to plot a $3 \mathrm{D}$ position, which is more accurate than $2 \mathrm{D}$ [11].

\subsubsection{Three Segments of GPS \\ 1. Space Segment \\ 2. Control Segment \\ 3. User Segment (see fig.1)}

Space segment: The satellites orbiting around the earth. Control segment: The control \& monitoring stations.

User Segment: The GPS receivers owned by civilians and military.

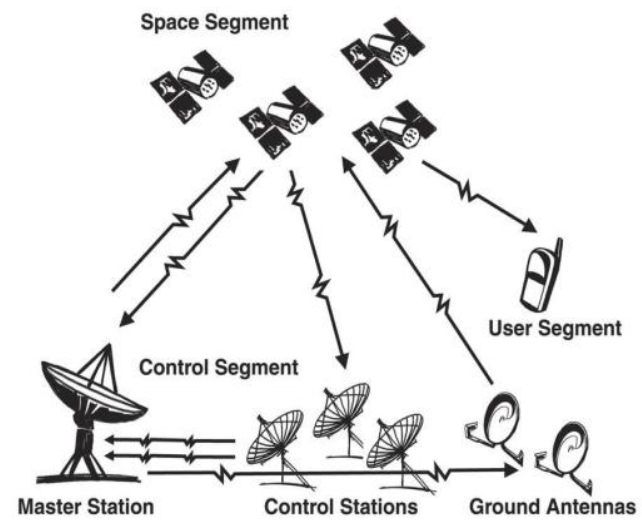

Fig. 1 Three segments of GPS [15]

In this paper we use the GPS in Dijkstra's algorithm for finding the current location. By using this position we calculate the distance from source to every node in the graph. From this we also estimate the shortest path. Distance is given by a formula:

$$
\text { Distance }=\left[\left(\mathrm{x}_{2}-\mathrm{x}_{1}\right)^{2}+\left(\mathrm{y}_{2}-\mathrm{y}_{1}\right)^{2}+\left(\mathrm{z}_{2}-\mathrm{z}_{1}\right)^{2}\right]^{1 / 2}
$$

Where $\mathrm{x}, \mathrm{y}, \mathrm{z}$ are the coordinates of a position given by GPS. In this paper we discussed about 1 is introduction, 2 is related work, 3 is proposed work, 4 is results and analysis, 5 is conclusion and future work \& 6is references.

\section{RELATED WORK}

GPS is used for tracking your vehicles \& keeps regular monitoring. This tracking system can tell your location \& that information can be observed from another remote location. This paper consists hardware part i.e. GPS,GSM,MAX $232,16 * 2$ LCD \&software part is used for interfacing all the required modules \& a web application is also developed at client side. This is used for controlling theft of a vehicle. The simulation is done by PROTEUS software. It can be beneficial for:

1. Parents to look after their children. 
2. To track animals in forest

3 . in delivery services

4. in fire services \& COP department [1].

We have read about the bus monitoring using polyline algorithm. Day by day population is increases very fastly, which results in higher burden on public transportation. For a system is proposed in this paper. This system is developed using these technologies like GPS, Google Map \& GPRS (Global Packet Radio Service) .the consists of GPS enabled device like mobile phones embedded in the bus, which find out its current coordinates periodically after some interval \& send it to the database for the being processed \& analyzed. Dijkstra's algorithm is also used in this paper [2].

The concept developed is focused on one of the most well known shortest path algorithm: the Dijkstra's algorithm. Although the latter is sufficiently efficient for small network like a city sized one, its running time for country size or continental size geographical maps is prohibitive for real time application. This method is also applicable to other type of shortest path algorithms on graph network. The basic algorithm for this is the Dijkstra's algorithm [3].

A route planning project named planific@ is developed for the city of Madrid (Spain).its main objective is to develop an intelligent system that is capable of routing people from one place to other using public transport. For this we study the route planning algorithm for this. In this Dijkstra's algorithm is also used for finding the shortest path. Planning Domain Definition Language (PDDL) is also used in this paper [4].

Location based services offer many benefits to mobile user to retrieve the information about their current location \& process that data to get more useful information near to their location. Using a GPS assisted phone \& a web service using GPRS ,location based services can be implemented on Android based smart phones to provide services like advising client of current traffic conditions, providing routing information, helping them find nearby hotels. In paper location based services is implemented through Google Web Services \& Walk Score Transit APIs on Android Phones to give multiple services to the user based on their location [5].

The Real Time GPS Navigation System determines the user's location on the digital map of Beirut using a GPS receiver. The user enters the destination and the shortest path is computed using Dijkstra's algorithm. The application guides the user along the way by giving him/her directions. The user can take a different route and the corresponding shortest path is dynamically recomputed. However, no matter how accurate the GPS is, errors may occur; the GPS fails to show the correct user's position. Corrections used in our application are based on pattern recognition techniques which take several previous GPS measurements to estimate the current position. Our Real Time GPS Navigation System was realized using innovative techniques. The shortest path algorithm, for example, is an improvement over Dijkstra's classical algorithm. The driving directions are generated by analyzing the geometry of the map instead of using a table at each node. Finally, the correction of the GPS errors and their matching on the map are performed without requiring any radio signals; they rely only on the raw GPS signal and the geometry of the road network. Moreover, the system has a user-friendly interface that facilitates traveling in the city of Beirut [6].

\section{PROPOSED WORK}

In this paper, we use the current position in the Dijkstra's algorithm using GPS. From this position we calculate the distance from source to that position. In this we find a shortest path from a sources to others vertices $\mathrm{v}$ in a graph. GPS is a satellite based system that is used in navigation, tracking \& mapping application. In this paper we use the concept of GPS in Dijkstra's shortest path algorithm for getting the current position of the nodes in the graph. In this paper we give only the theoretical ideas about this process. No implementation \& practical data is given in this paper. We proposed a model \& an algorithm for this.

\subsection{Proposed Model}

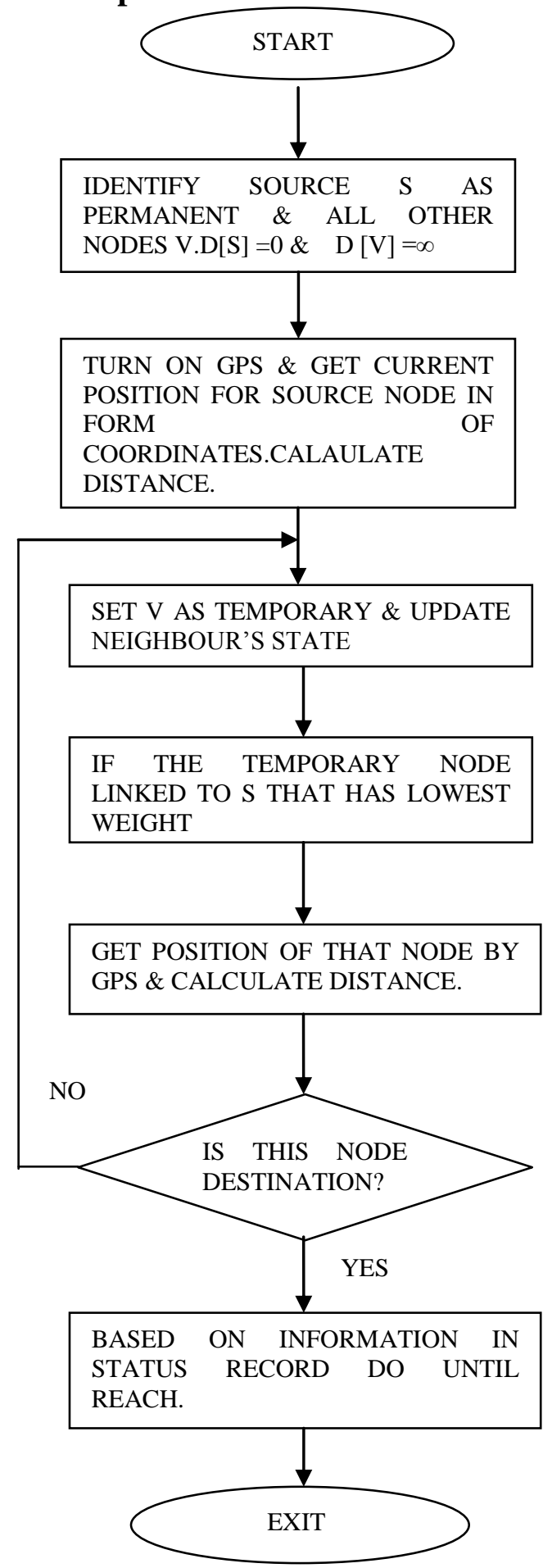

Fig. 2 Proposed Model 


\subsection{Proposed Algorithm}

Step1: INITIALIZE $\mathrm{d}[\mathrm{s}]=0$ for all $\mathrm{veV}$

$\{\mathrm{s}\}$, where $\mathrm{s}$ as source, $\mathrm{V}$ as set of all vertices.

$$
\text { Do } \mathrm{d}[\mathrm{v}]=\infty \text {. }
$$

$/ /$ set all node's distances to $\infty$ except $\mathrm{s}$.

Step2: Get the current position $\left(\mathrm{x}_{1}, \mathrm{y}_{1}\right)$ of source node from GPS

Source_x $=\mathrm{x}_{1}$;

Source_y $=\mathrm{y}_{1}$;

Dist. $=0$;

Step3: $S$ is the set of visited vertices.
Set $S=\varphi$
//S is initially empty.

$$
\mathrm{Q}=\mathrm{V}
$$
//Queue initially contain all the

vertices.

While $\mathrm{Q} \neq \varphi$

//while Q is

not initially empty.

Do $\mathrm{u}=$ mindistance $(\mathrm{Q}, \mathrm{d}) \quad / /$ select element of $\mathrm{Q}$ with min. distance.

$\mathrm{S}=\mathrm{S} \mathrm{U}\{\mathrm{u}\} \quad$ //add $\mathrm{u}$ to the list of visited vertices.

Step4: Get the position $\left(\mathrm{x}_{2}, \mathrm{y}_{2}\right)$ of the

visited nodes from GPS

Current_x $=\mathrm{x}_{2}$;

Current_y $=\mathrm{y}_{2}$;

distance $=\sqrt{ }\left(\mathrm{x}_{2}-\text { source } \_\mathrm{x}\right)^{2}+\left(\mathrm{y}_{2}-\right.$

source_y $)^{2}$

dist $=$ distance + dist

Previous $\mathrm{x}=\mathrm{x}_{2}$

Previous_y $=\mathrm{y}_{2}$;

Step5: For all $v \in$ neighbors[u]

Do if $d[v]>d[u]+w[u, v] \quad$ //if new shortest path found.

Then

$\mathrm{d}[\mathrm{v}]=\mathrm{d}[\mathrm{u}]+\mathrm{w}[\mathrm{u}, \mathrm{v}]$

//see new value of shortest path if desired then trackback.

Return dis.

Step6: Get the position $\left(\mathrm{x}_{2}, \mathrm{y}_{2}\right)$ of the visited nodes from GPS

Current_x $=\mathrm{x}_{2}$;

Current_y $=\mathrm{y}_{2}$; source y) ${ }^{2}$

distance $\left.=\sqrt{\left(x_{2}-\text { source }\right.} \quad \mathrm{x}\right)^{2}+\left(\mathrm{y}_{2}-\right.$

dist $=$ distance + dist

Previous_x $=\mathrm{x}_{2}$;

Previous_y $=\mathrm{y}_{2}$;

\subsection{Example:}

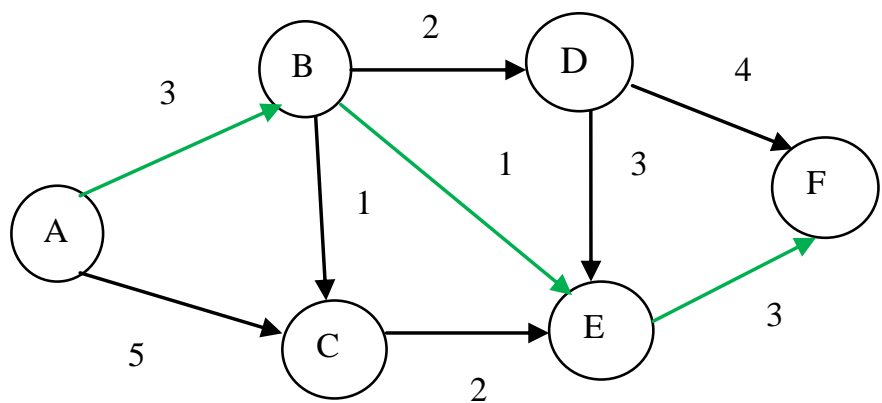

Fig. 3 Example for shortest path
Table1. For finding the shortest path using Dijkstra's \&

\begin{tabular}{|c|c|c|c|c|c|c|c|}
\hline \multirow[t]{2}{*}{ STEPS } & \multirow{2}{*}{$\begin{array}{c}N \\
1\{A\}\end{array}$} & \multicolumn{6}{|c|}{ POSITION DISTANCE D(B),PATR D(C),PATK D(D),PATK D(E),PATH D(F),PATH } \\
\hline & & $(2,3)$ & 0 3,A-B & 5,A-C & $\infty,-$ & $\infty,-$ & $\infty,-$ \\
\hline & $2\{A, B\}$ & $(5,7)$ & 5 3,A-B & 4,A-B-C & 5,A-B-D & 4,A-B-E & $\infty,-$ \\
\hline & $3\{A, B, C\}$ & $(8,11)$ & $103, A-B$ & 4,A-B-C & 5,A-B-D & 4,A-B-E & $\infty,-$ \\
\hline & $4\{A, B, C, E\}$ & $(11,15)$ & 15 3,A-B & 4,A-B-C & 5,A-B-D & 4,A-B-E & 7,A-B-E-F \\
\hline & $5\{A, B, C, D, E$ & $(14,19)$ & 20 3,A-B & 4,A-B-C & 5,A-B-D & 4,A-B-E & 7,A-B-E-F \\
\hline & $6\{\mathrm{~A}, \mathrm{~B}, \mathrm{C}, \mathrm{D}, \mathrm{E}$ & $(17,23)$ & 25 3,A-B & 4,A-B-C & 5,A-B-D & 4,A-B-E & 7,A-B-E-F \\
\hline
\end{tabular}
GPS

\section{RESULT \& ANALYSIS}

In this section, we give the results by using the MATLAB .we represents the results in the form of graphs. We use the fuzzy logic in the MATLAB. We are using the rules for this.

\section{USING DIJKSTRA ALGORITHM}

TABLE 2. Impact of Distance on Path

\begin{tabular}{|l|l|}
\hline DISTANCE & SHORTEST_PATH \\
\hline Min. & Selected \\
\hline Max. & Not Selected \\
\hline
\end{tabular}

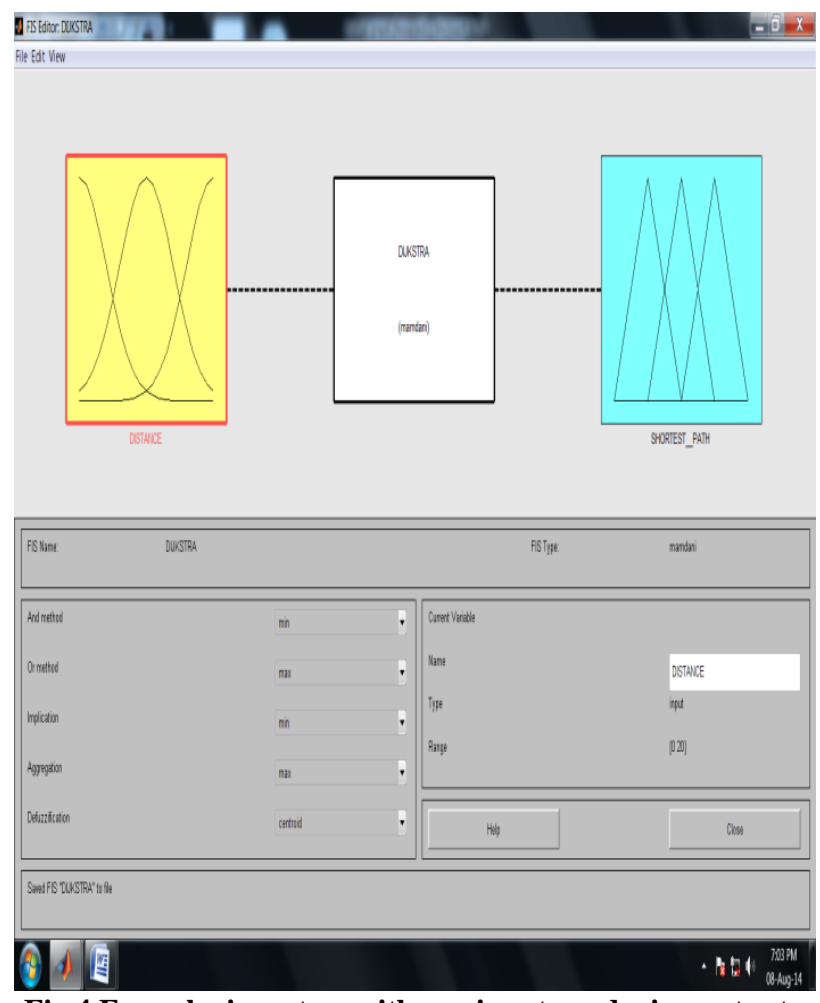

Fig.4 Fuzzy logic system with one input producing output as shortest path selected 


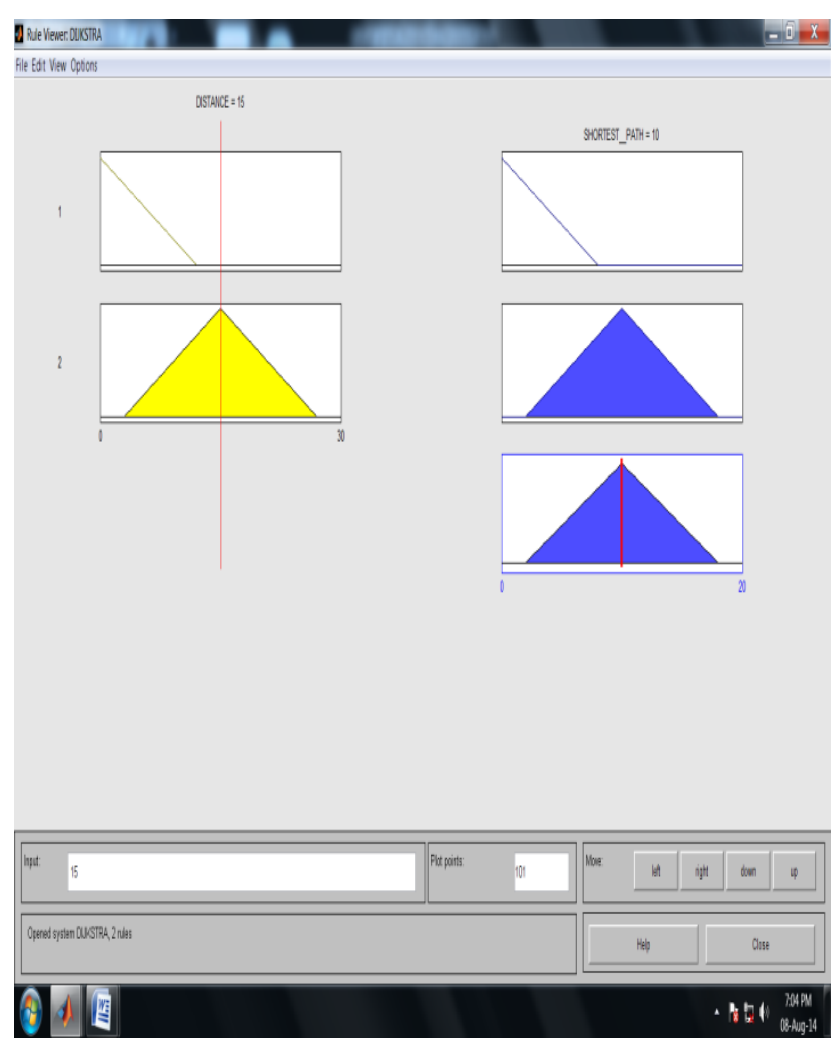

Fig.5 Fuzzy logic rule based viewer

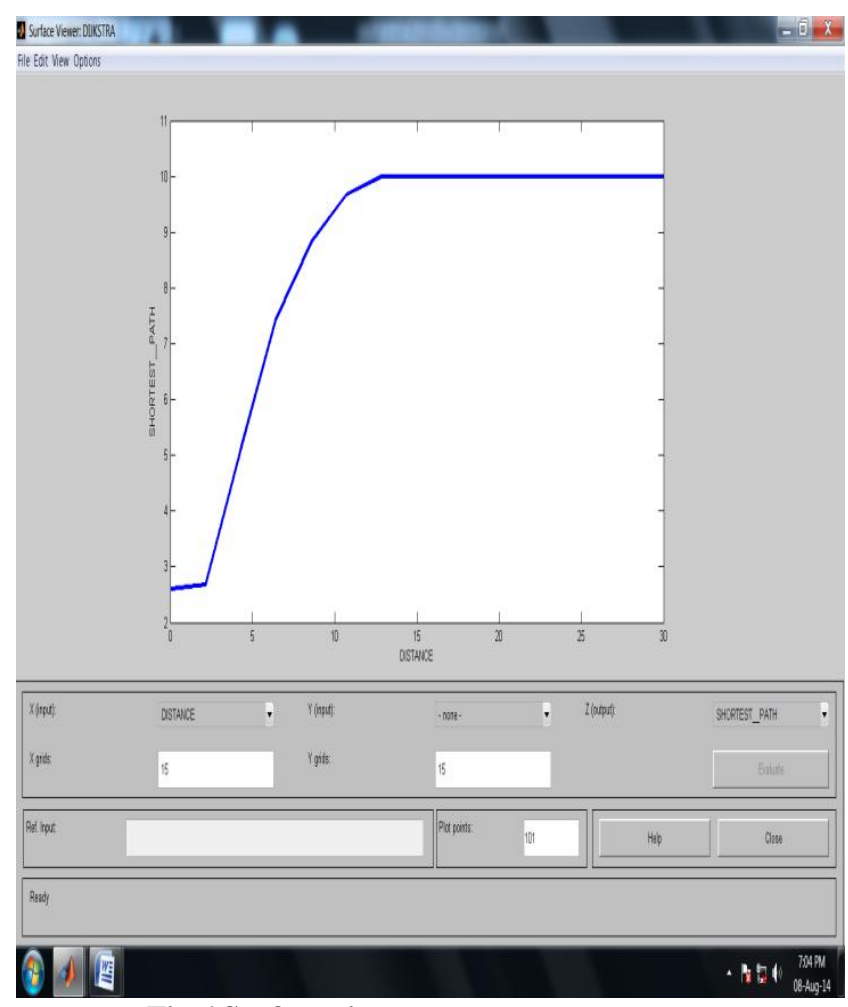

Fig.6 Surface viewer

\section{USING GPS \& DIJKSTRA}

TABLE3. Impact of Distance \& Position on Path

\begin{tabular}{|l|l|l|}
\hline DISTANCE & POSITION & SHORTEST_PATH \\
\hline Min. & Accurate & Selected \\
\hline Max. & $\begin{array}{l}\text { Not } \\
\text { Accurate }\end{array}$ & Not Selected \\
\hline
\end{tabular}

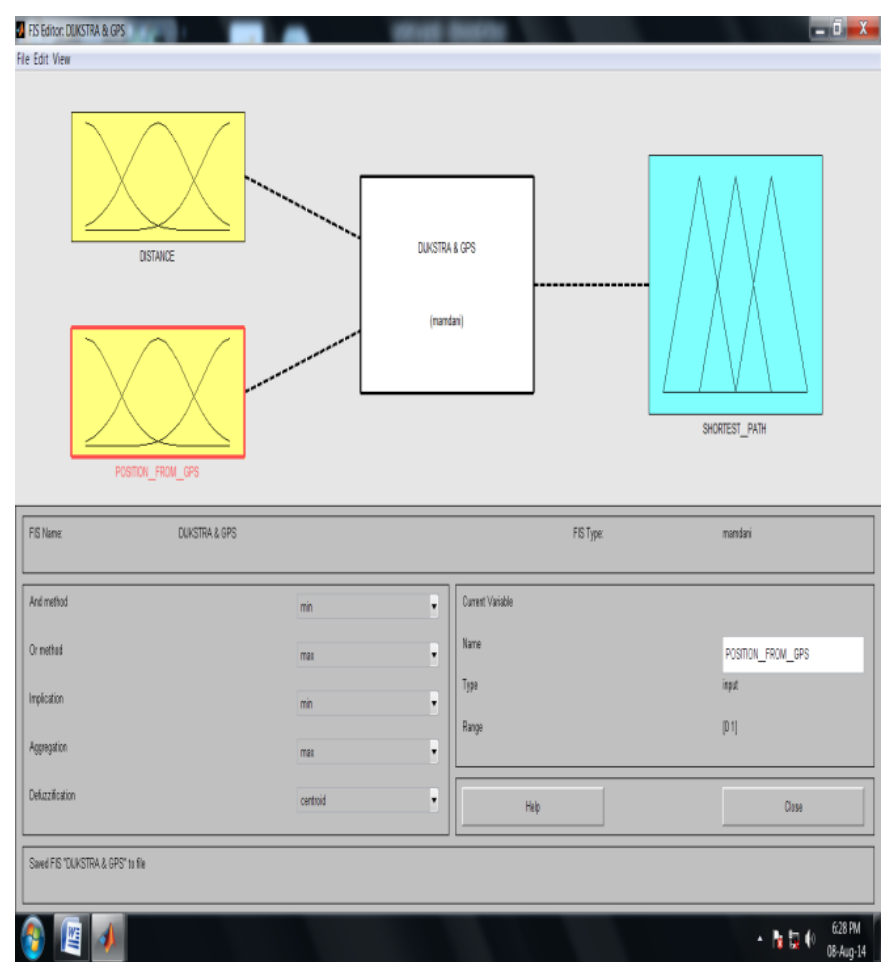

Fig.7 Fuzzy logic system with two input \& producing shortest path as output

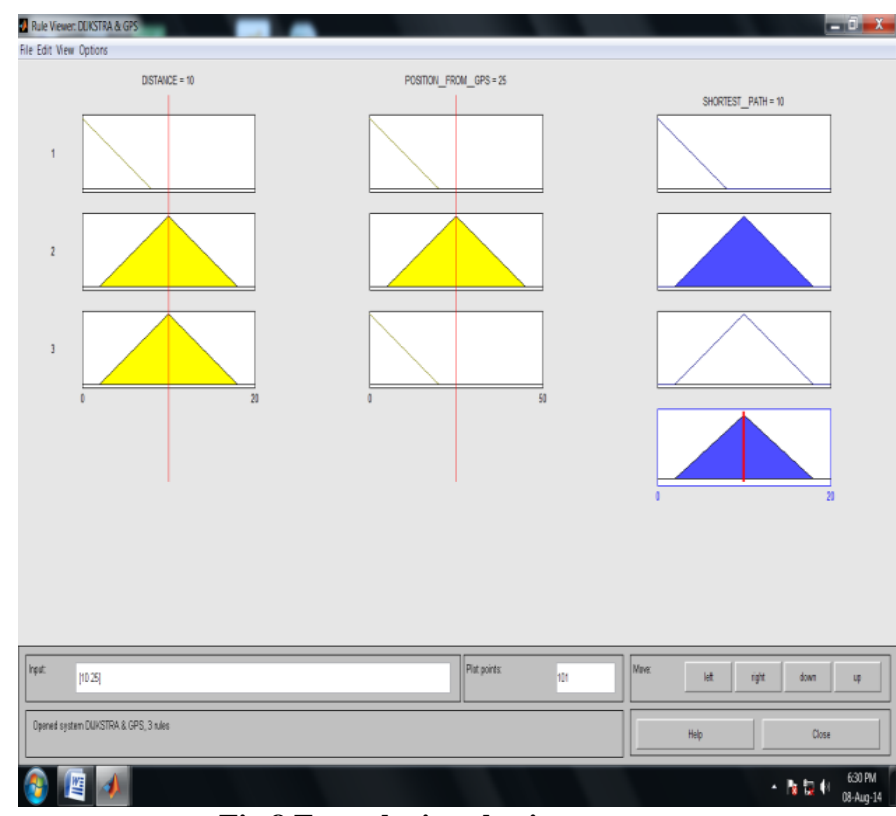

Fig.8 Fuzzy logic rule viewer 


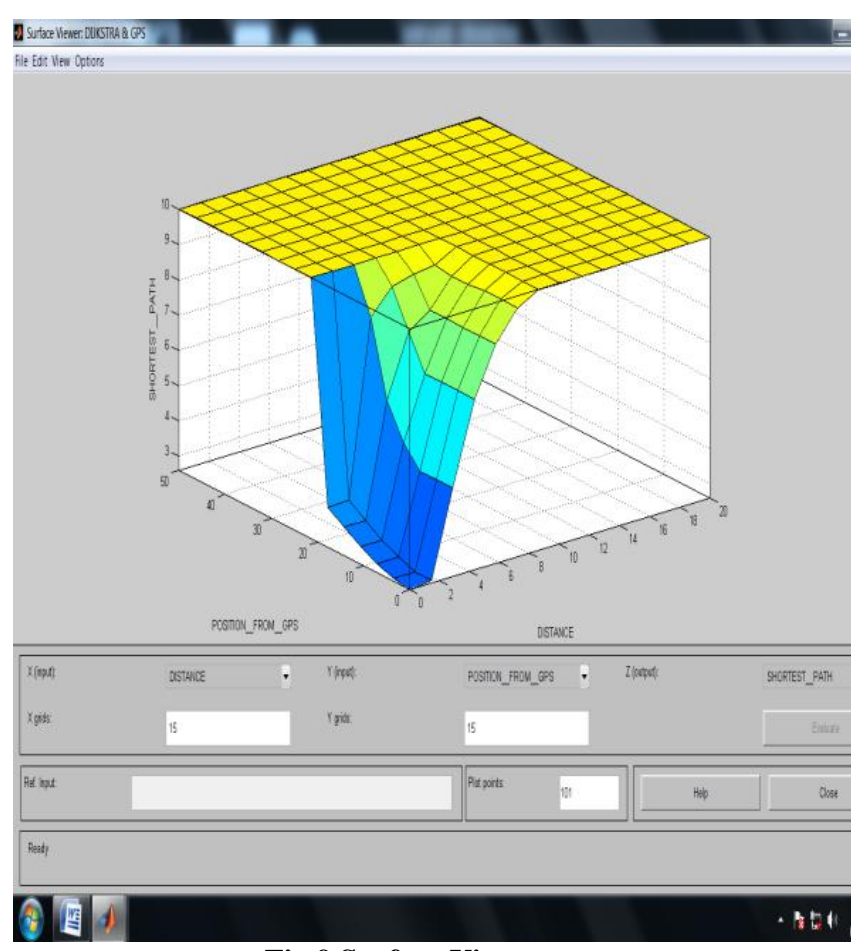

Fig.9 Surface Viewer

\section{USING POSITION \& ROUTE}

TABLE4. Impact Of Position On Route

\begin{tabular}{|l|l|}
\hline POSITION & ROUTE \\
\hline Accurate & Selected \\
\hline Not Accurate & Not Selected \\
\hline
\end{tabular}

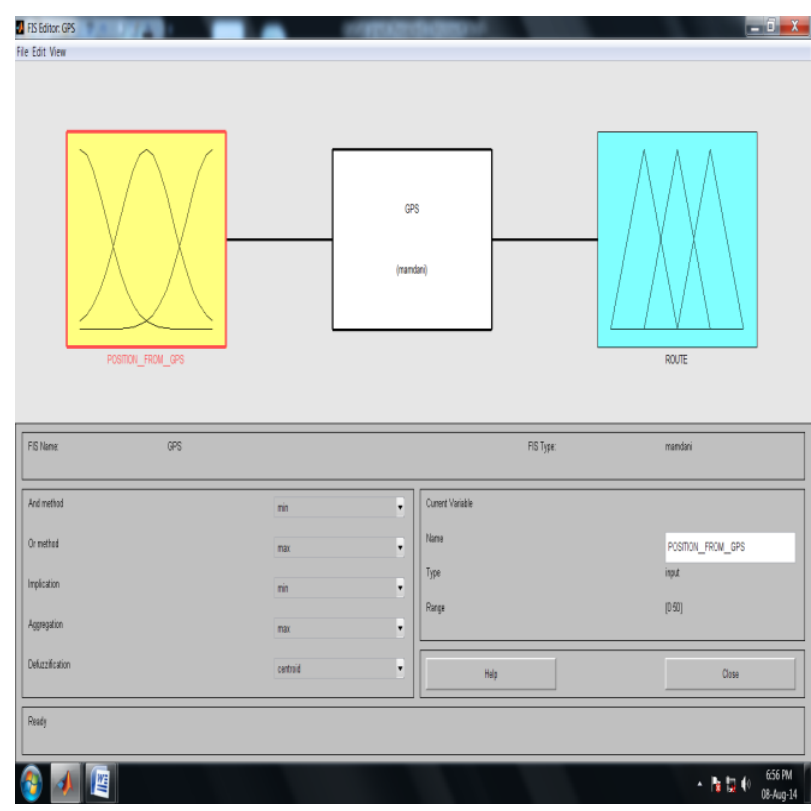

Fig.10 Fuzzy logic system with one input \& producing output route selected

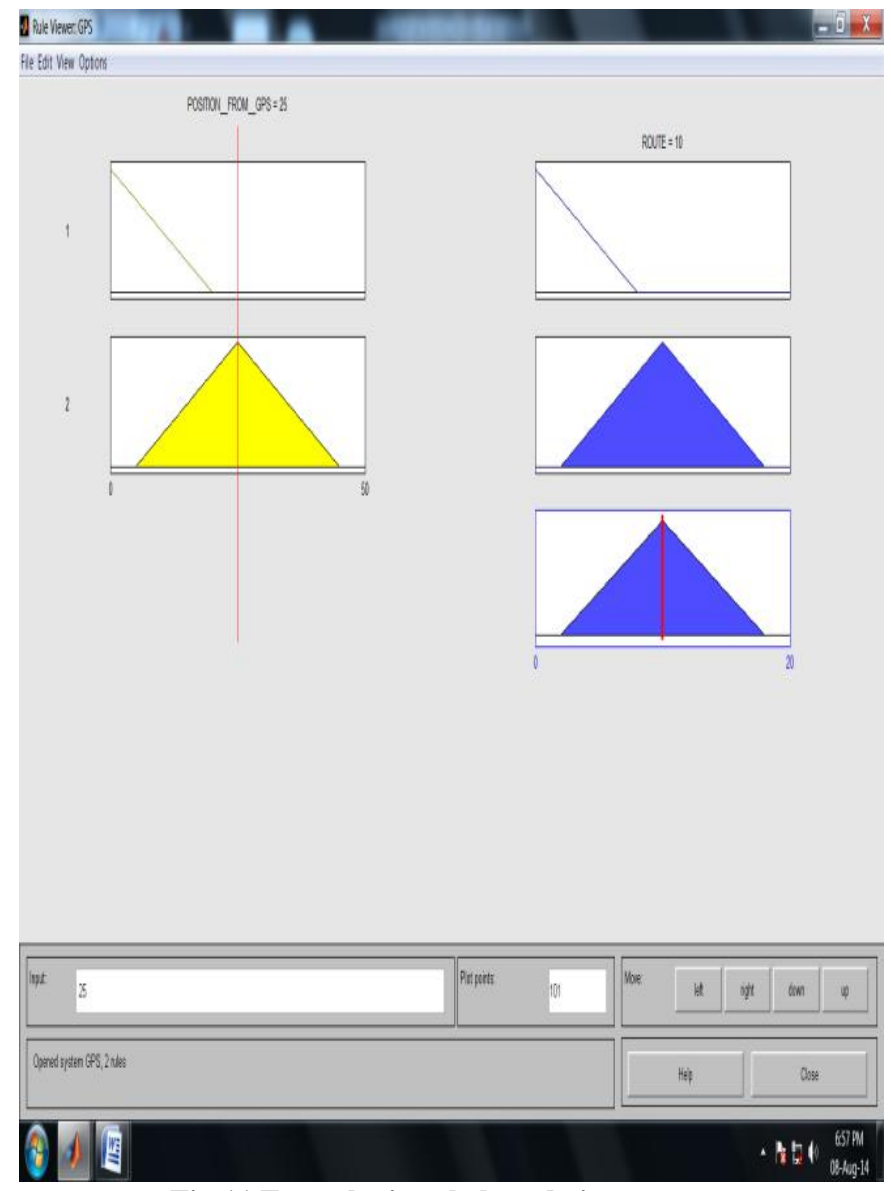

Fig.11 Fuzzy logic rule based viewer

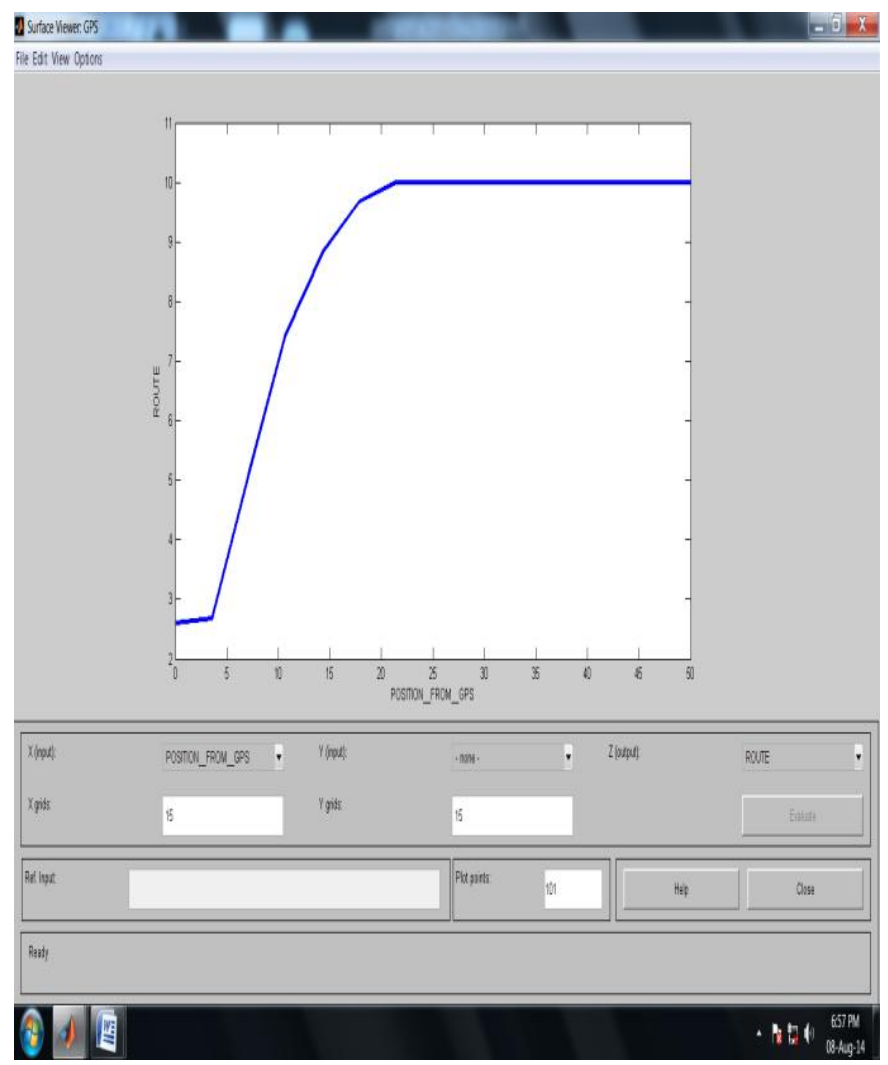

Fig.12 Surface Viewer 
USING TIME \& PATH

Table5. Impact of Distance \& Time on Path

\begin{tabular}{|l|l|l|}
\hline DISTANCE & TIME & SHORTEST_PATH \\
\hline Min. & Less & Selected \\
\hline Max. & More & Not Selected \\
\hline Min. & More & Not Selected \\
\hline
\end{tabular}

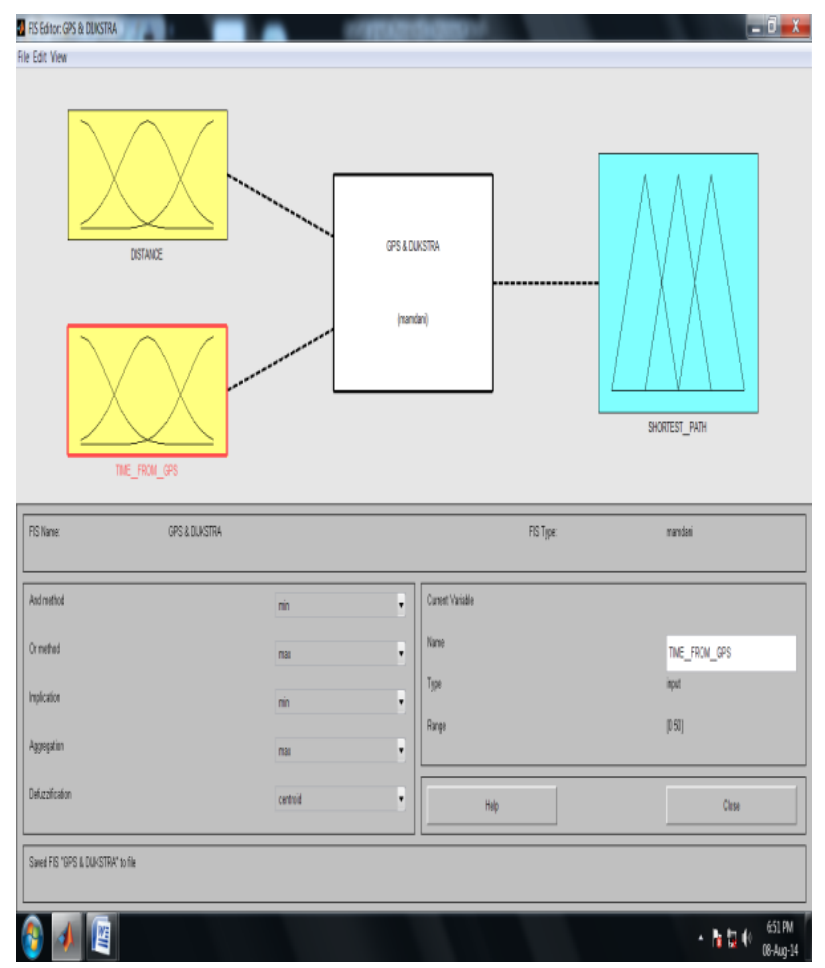

Fig.13 Fuzzy logic system with two input \& producing shortest path as output

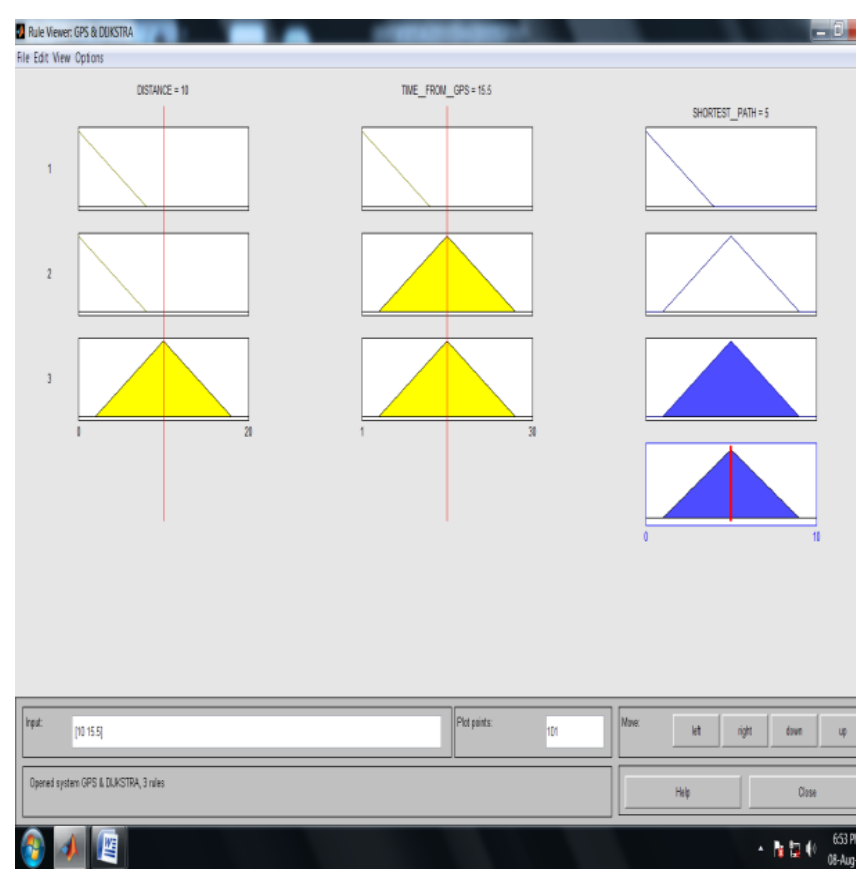

Fig.14 Fuzzy logic rule based viewer

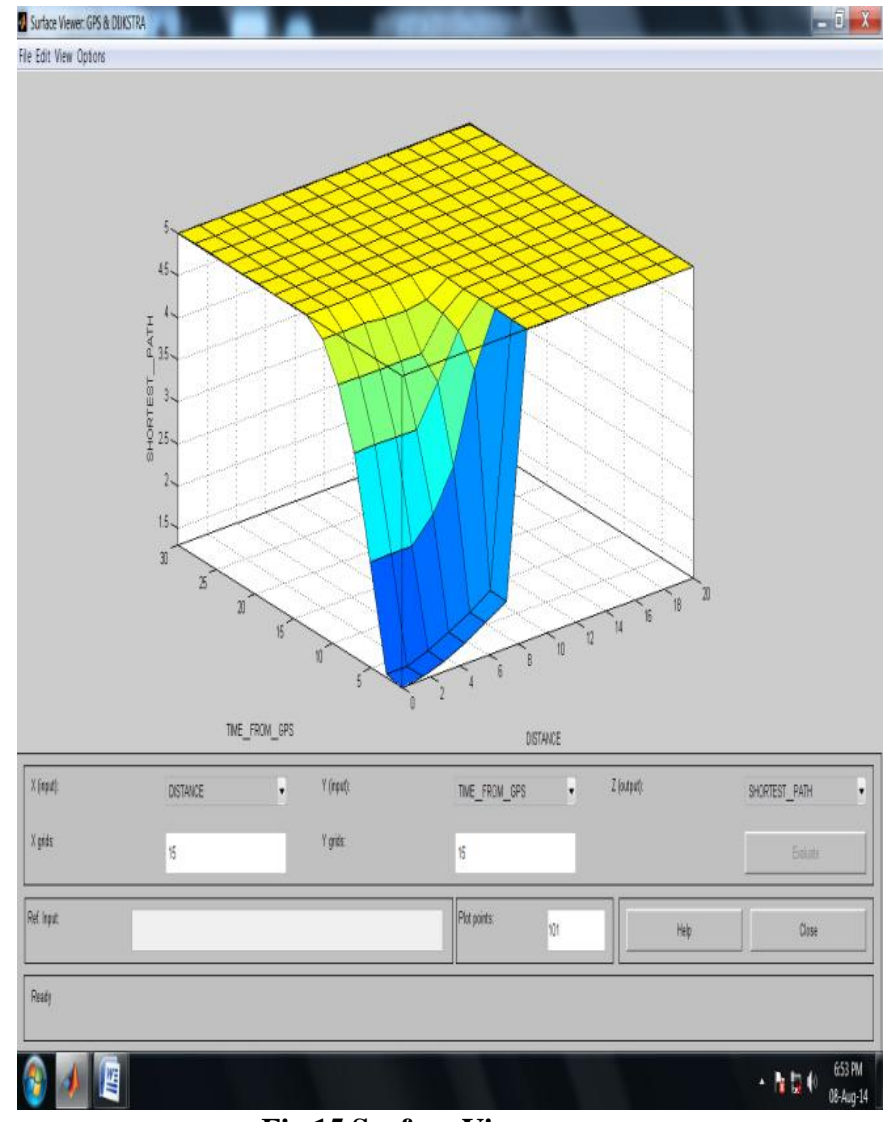

Fig.15 Surface Viewer

\section{CONCLUSION \& FUTURE SCOPE}

In this paper Dijkstra's algorithm is used to find out the shortest path in a graph. GPS is used in Dijkstra's algorithm to get the current position of each node. Distance is also calculated from this position. An algorithm is proposed for this. But only theoretical concept is given, practical implementation is not given in this paper. So, in future you can implement this algorithm practically. This concept of GPS is also used on others shortest path algorithms like $A^{*}$, Warshals algorithm \& Bellman Ford algorithm etc.

\section{REFERENCES}

[1] Pankaj Verma , J.S Bhatia, "Design And Development Of GPS-GSM Based Tracking System With Google Map Based Monitoring", International Journal of Computer Science, Engineering and Applications (IJCSEA) Vol.3, No.3, June 2013.

[2] Vishal Bharte, Kaustubh Patil, Lalit Jadhav, Dhaval Joshi, "Bus Monitoring System Using Polyline Algorithm", International Journal of Scientific and Research Publications, Volume 4, Issue 4, April 2014.

[3] Sacha Varone, "On a many-to-one shortest paths for a taxi Service", Haute ecole de gestion de Gen eve CRAG Centre de Recherché Appliqué ee en Gestion Cahier de Recherché

[4] Carlos Martín García and Gonzalo Martín Ortega, "Route planning algorithms: Planific@ Project”, International Journal of Artificial Intelligence and Interactive Multimedia, Vol. 1, No 2.

[5] Manav Singhal, Anupam Shukla, "Implementation of Location based Services in Android using GPS and Web 
Services", IJCSI International Journal of Computer Science Issues, Vol. 9, Issue 1, No 2, January 2012.

[6] Abboud, Marwan, LM Abou Jaoude, and Ziad Kerbage. "Real Time GPS Navigation System." disponible sur http://webfea-lb. fea. aub. edu. lb/proceedings/2004/SRCECE-27. pdf (2004).

[7] A.Prakash, R.Manickavasagam, "Elegant Way of Reaching Destination Using GPS and Drivers Ability", International Journal of Advanced Research in Computer Science and Software Engineering, Volume 4, Issue 3, March 2014.
[8] Hu Jian-ming; Li Jie; Li Guang-Hui, "Automobile Antitheft System Based on GSM and GPS Module," Intelligent Networks and Intelligent Systems (ICINIS), 2012 Fifth International Conference on , vol., no., pp.199,201, 1-3 Nov. 2012

[9] PMS475_chap5.pdf

[10] 1-Melissa.pdf

[11]http://4.bp.blogspot.com/HqYRvKnZe1k/UIQi0QkA1WI/ AAAAAAAAABo/Tc1UUf84Ok8/s1600/gps_segments\% 5B1\%5D.jpg 